\title{
Influenza hospitalizations in Australian children
}

\author{
J. LI-KIM-MOY ${ }^{1,2,3 *}$, J. K. YIN ${ }^{2,3}$, C. C. BLYTH ${ }^{4,5,6}$, A. KESSON ${ }^{1,3}$, \\ R. BOOY ${ }^{1,2,3}$, A. C. CHENG $^{7,8}$ AND K. MACARTNEY ${ }^{1,2,3}$ \\ ${ }^{1}$ The Children's Hospital at Westmead, Sydney, Australia \\ ${ }^{2}$ National Centre for Immunisation Research and Surveillance, Sydney, Australia \\ ${ }^{3}$ University of Sydney, New South Wales, Australia \\ ${ }_{5}^{4}$ School of Paediatrics and Child Health, University of Western Australia, Australia \\ ${ }^{5}$ Departments of Infectious Diseases and Microbiology, Princess Margaret Hospital, Perth, Western Australia \\ ${ }^{6}$ Wesfarmers Centre for Vaccines and Infectious Diseases, Telethon Kids Institute, Western Australia \\ ${ }^{7}$ Infection Prevention and Healthcare Epidemiology Unit, Alfred Health, Melbourne, Victoria, Australia \\ ${ }^{8}$ Department of Epidemiology and Preventive Medicine, Monash University, Victoria, Australia
}

Received 27 September 2016; Final revision 13 November 2016; Accepted 16 December 2016; first published online 6 February 2017

\section{SUMMARY}

Australia's National Immunisation Program (NIP) provides free influenza vaccination for children at high risk of severe influenza; a pilot-funded programme for vaccine in all children aged 6 months to $<5$ years in one of eight states, has seen poor vaccine impact, related to recent vaccine safety concerns. This retrospective review examined influenza hospitalizations in children aged $<16$ years from three seasons (2011-2013) at two paediatric hospitals on opposite sides of the country. Comparisons of this cohort were made with state-based data on influenza-coded hospitalizations and national immunization register data on population-level immunization coverage. Of 740 hospitalizations, the majority were aged $<5$ years $(476 / 740,64 \%)$, and a substantial proportion $(57 \%)$ involved healthy children, not currently funded for influenza vaccine. Intensive care unit admission occurred in $8.5 \%$, and $1.5 \%$ of all children developed encephalitis. Use of antiviral therapy was uncommon $(20.5 \%)$ and decreasing. Of those hospitalized, only $5.0 \%$ of at-risk children, who are currently eligible for free vaccine, and $0.7 \%$ of healthy children were vaccinated prior to hospitalization. This was consistent with low population-wide estimates of influenza vaccine uptake. It highlights the need to examine alternative strategies, such as universally funded paediatric influenza vaccination, to address disease burden in Australian children.

Key words: Children, epidemiology, hospitalization, Influenza, vaccination.

\section{INTRODUCTION}

Influenza is a respiratory infection that is the most frequently reported notifiable disease and a common

\footnotetext{
* Author for correspondence: Dr J. Li-Kim-Moy, National Centre for Immunisation Research \& Surveillance (NCIRS), Kids Research Institute, The Children's Hospital at Westmead, Corner Hawkesbury Road and Hainsworth Street, Westmead, NSW 2145, Australia. (Email: jean.likimmoy@health.nsw.gov.au)
}

cause of hospitalizations each year in Australia. Hospitalization rates during 2005-2008 in children aged $<5$ years $(48-65 / 100000)$ exceeded those in the elderly aged $>65$ years $(13 \cdot 4-20 / 100000$ annually) [1-3]. The highest hospitalization rates are in those aged $<6$ months $(192-270 / 100000)$, a group potentially amenable to protection through maternal vaccination during pregnancy $[3,4]$. 
For children and young adults, Australia has 'targeted' influenza vaccination funded under the National Immunisation Program (NIP). Since 2010, free NIP-funded vaccine has been available for high-risk individuals with chronic medical conditions aged $\geqslant 6$ months, pregnant women, and Indigenous children aged $\geqslant 15$ years $[5,6]$. A funded influenza immunization programme for all children aged 6 months to $<5$ years exists in only one state (Western Australia; WA), introduced in 2008 after three deaths in healthy children in a single season [7]. However, following an unexpected increase in febrile seizures with one brand of trivalent influenza vaccine (TIV) in Australian children aged $<5$ years during $2010[8,9]$, the majority of which were documented in WA due to higher uptake with its funded vaccination programme (then $>40 \%$ ), public confidence was affected; vaccine uptake fell precipitously to $<10 \%$ [10] and remains low despite the safety of other brands being confirmed $[11,12]$. This contrasts with universal vaccination programmes in the United States, Canada, and UK where coverage rates have increased up to $60 \%$ of eligible children [13-18].

Uncertainty remains as to how well Australia's targeted strategy is working and whether at-risk children are being adequately protected through vaccination. Data on vaccine uptake in those with underlying medical conditions is scarce. One small study conducted in 2012 found that coverage in at-risk children attending outpatient specialist clinics was $41 \%$ but relied on unverified parental report in this select population [19]. Previous epidemiological data [1] regarding severe influenza in Australia have relied on administrative hospitalization data, without clinical confirmation; this provides no detailed information on who is affected or vaccine uptake [20]. Other small studies have been limited to examining a single season or centre [21, 22]. To guide future prospective surveillance aimed at assessing annual influenza disease burden and vaccine effectiveness against influenza hospitalization in children and adults [23], we conducted a retrospective examination of influenza-related hospitalizations in two large paediatric hospitals during 2011-2013, to describe the demographic and clinical characteristics and outcomes of severe influenza. The data would also help to better understand the pattern of hospitalization in children and to inform influenza surveillance and vaccination strategies.

\section{MATERIALS AND METHODS}

This was a retrospective review of influenza hospitalizations in children aged $<16$ years, over a 3 -year period from January 2011 to December 2013 in two major centres: the Children's Hospital at Westmead (CHW), Sydney, New South Wales (NSW) and the Princess Margaret Hospital for Children (PMH), Perth, WA, which are part of the Flu Complications Alert Network (FluCAN) [24], a sentinel network of 17 hospitals collecting data on adult and paediatric influenza hospitalizations each season. CHW and PMH function as both general paediatric hospitals for a proportion of each city's population, as well as tertiary referral centres within their respective states. Cases were obtained from each hospital's laboratory database. A case was included if they were a hospitalized child who had laboratory-confirmed influenza by polymerase chain reaction (PCR) for A and B viruses (within a multiplex respiratory virus panel), at presentation or during their hospital stay, and an acute respiratory infection (ARI), defined as symptoms or signs of upper or lower respiratory tract infection (e.g. cough, coryza, sore throat, shortness of breath, wheeze, or tachypnoea), with or without fever during the study period. Diagnoses of influenza only by direct fluorescent antibody testing or point-of-care testing were excluded. In both hospitals, it is routine practice, but at the physician's discretion, for most hospitalized children with ARI to undergo respiratory virological testing for diagnostic purposes and to facilitate infection control.

Demographic data, medical history (including comorbidities), clinical symptoms and signs at presentation (fever, respiratory distress, or other symptoms), investigations performed [chest X-ray, blood culture, lumbar punctures (LP), influenza virus subtyping], and management, including use of antivirals and antibiotics were recorded. Conditions predisposing to severe influenza infection (as listed in the Australian Immunisation Handbook [25]) were documented. Comparisons were made between seasons and according to influenza type. Pneumonia was defined as consolidation recorded on chest X-ray (CXR) report. Bacterial infection included meningitis, bacteraemia, urinary tract infection, or other sterile site infection where a bacterial pathogen was identified on an appropriate specimen by culture and/or antigen testing. We assessed outcomes including length of stay (LOS) in hospital, intensive care unit (ICU) admission, associated bacterial infection, seizures, encephalitis, or in-hospital death.

Numbers of hospitalizations at each site were compared with de-identified state-wide hospitalization data obtained from the WA and NSW Departments 
of Health for children aged 0-18 years with ICD-10-AM coded influenza (J09-J10) as a primary or secondary diagnosis.

Influenza vaccination status (including the years prior to and subsequent to hospitalization) was assessed for the included hospitalized children or their mothers (if child aged $<6$ months) from both the medical record and the Australian Childhood Immunisation Register (ACIR), which records immunizations for all children aged $<7$ years [26]. In addition, we obtained population-level influenza vaccine coverage during the study period for all children contained within ACIR at the national and state levels.

Differences between groups were assessed using $\chi^{2}$ test for categorical variables and independent-sample $t$ tests or Mann-Whitney $U$ test for continuous variables. We used multivariate logistic regression to examine for risk factors associated with ICU admission. Predictors with $P<0.20$ on univariate analysis were incorporated into a stepwise regression model with backwards elimination to determine adjusted odds ratios. For hospital LOS, the distribution was expected to be substantially positively skewed, therefore we used a generalized linear model with negative binomial distribution for multivariate regression analysis. Adjusted ratios of mean LOS were calculated for predictor variables from the final multivariate model. Calculations were performed using SPSS Statistics v. 21 (IBM Corp., USA).

Ethical approval was obtained for this research from the Sydney Children's Hospitals Network Human Research Ethics Committee (reference HREC/13/SCHN/402).

\section{RESULTS}

During the study period, 740 PCR-confirmed influenza hospitalizations occurred (Table 1). The majority were during the July-September winter influenza season, typical for the temperate climate locations. Overall, $65 \%(n=480)$ of cases were admitted to CHW and $35 \%(n=260)$ to PMH. This represented $23 \%(480 / 2075)$ and $41 \%(260 / 634)$ of all ICD-coded influenza hospitalizations during the 3 years in NSW (annual birth cohort: 99000 ) and WA (annual birth cohort: 33000), respectively [27]. Males comprised a larger percentage of hospitalizations $(55.5 \%, P=0.003)$. Young children were overrepresented; 64\% (476/740) were aged $<5$ years; $13.4 \%(n=99)$ of the total were aged $0-<6$ months (Fig. 1). Indigenous children accounted for $4 \cdot 2 \%$ of all admissions. Many children $(43 \%, n=317)$ had one or more medical conditions predisposing to severe influenza infection, most commonly immunosuppression $(13 \cdot 2 \%)$, neurological disease $(12 \cdot 7 \%)$ and chronic respiratory disease $(10 \cdot 4 \%)$. There were no significant differences between $\mathrm{CHW}$ and $\mathrm{PMH}$ in terms of age distribution or proportion of children with underlying medical conditions; median LOS was slightly shorter at PMH (2 days) than CHW (3 days).

\section{Influenza vaccination}

In hospitalized children included in our study, who were aged 6 months -7 years and had data captured by the ACIR, 2.5\% (12/474) had at least one influenza vaccination $>2$ weeks prior to hospitalization and in the same year. The rate of vaccination was greater in children at the WA site compared to NSW (4.3\% vs. $1.6 \%$ ). A higher proportion of children aged 6 months-7 years $(10 \cdot 8 \%, 51 / 474)$ were recorded as receiving an influenza vaccination in the year following their hospitalization. No children had data recorded in their clinical record regarding receipt of vaccination, including no record of maternal vaccination (for those aged $<6$ months). Population-wide estimates from ACIR revealed similarly low vaccination rates in all children within NSW $(0 \cdot 5-1 \cdot 0 \%)$, WA $(3 \cdot 9-5 \cdot 1 \%)$, and nationally $(1 \cdot 0-1 \cdot 6 \%)$ during 2011-2013 (Table 2).

\section{Influenza types/subtypes}

Influenza A was the predominant type in 2011 (64.6\%) and 2012 (69\%). In 2013, both influenza A and B were equally common (Table 1). The median age was significantly higher for children with influenza B compared to influenza A $(5.0$ vs. 2.4 years, $P<0.001)$. Limited influenza A subtyping was available, predominantly at PMH (see Table 1). Influenza A or B strains were not associated with any differences in clinical features or outcomes except for myositis which was more common with influenza B infection $(P=0 \cdot 03)$.

\section{Clinical presentation}

Overall $75 \%$ of hospitalized patients with influenza presented with influenza-like illness (fever and cough and/or sore throat) [28] (Table 1). Fewer children presented with respiratory distress/pneumonia $(19 \cdot 1 \%)$ or fever without source (most common in neonates; $19 \%$ of whom presented in this way). Urinary tract infection 
Table 1. Demographic information, influenza strain, clinical presentation, and investigations by year in Australian children hospitalized with laboratory-confirmed influenza during 2011-2013 at the Children's Hospital at Westmead (NSW, Australia) and Princess Margaret Hospital (WA, Australia)

\begin{tabular}{|c|c|c|c|c|}
\hline & \multicolumn{3}{|l|}{ Year } & \multirow[b]{2}{*}{ Total } \\
\hline & 2011 & 2012 & 2013 & \\
\hline Total $(n)$ & 199 & 312 & 229 & 740 \\
\hline \multicolumn{5}{|l|}{ Sites } \\
\hline Children's Hospital at Westmead & $130(65 \cdot 3)$ & $166(53 \cdot 2)$ & $184(80 \cdot 3)$ & $480(64 \cdot 9)$ \\
\hline Princess Margaret Hospital & $69(34 \cdot 7)$ & $146(46 \cdot 8)$ & $45(19 \cdot 7)$ & $260(35 \cdot 1)$ \\
\hline \multicolumn{5}{|l|}{ Demographics } \\
\hline Male & $116(58 \cdot 3)$ & $168(53 \cdot 8)$ & $127(55 \cdot 5)$ & $411(55 \cdot 5)^{*}$ \\
\hline Median age, years & $3 \cdot 2$ & $3 \cdot 4$ & $2 \cdot 8$ & $3 \cdot 1$ \\
\hline \multicolumn{5}{|l|}{ Age groups ( $\%$ of year) } \\
\hline $0-28$ days & 6 & 4 & 6 & $16(2 \cdot 2)$ \\
\hline 29 days $-<6$ months & 20 & 35 & 28 & $83(11 \cdot 2)$ \\
\hline 6 months $-<1$ year & 24 & 34 & 22 & $80(10 \cdot 8)$ \\
\hline $1-<5$ years & 72 & 125 & 100 & $297(40 \cdot 1)$ \\
\hline $5-<10$ years & 50 & 82 & 53 & $185(25 \cdot 0)$ \\
\hline $10-15$ years & 27 & 32 & 20 & $79(10 \cdot 7)$ \\
\hline Indigenous & $12(6 \cdot 0)$ & $14(4 \cdot 5)$ & $5(2 \cdot 2)$ & $31(4 \cdot 2)$ \\
\hline Comorbidity present $\dagger$ & $90(45 \cdot 2)$ & $122(39 \cdot 1)$ & $105(45 \cdot 9)$ & $317(42 \cdot 8)$ \\
\hline Respiratory & 19 & 32 & 26 & $77(10 \cdot 4)$ \\
\hline Prematurity & 19 & 22 & 27 & $68(9 \cdot 2)$ \\
\hline Cardiac & 14 & 22 & 12 & $48(6 \cdot 5)$ \\
\hline Diabetes & 0 & 3 & 4 & $7(0 \cdot 9)$ \\
\hline Neurological & 29 & 35 & 30 & $94(12 \cdot 7)$ \\
\hline Renal & 9 & 6 & 11 & $26(3 \cdot 5)$ \\
\hline Immunosuppression & 30 & 38 & 30 & $98(13 \cdot 2)$ \\
\hline Hepatic & 7 & 7 & 4 & $18(2 \cdot 4)$ \\
\hline Genetic & 15 & 20 & 16 & $51(6 \cdot 9)$ \\
\hline Inborn error of metabolism & 4 & 5 & 5 & $14(1.9)$ \\
\hline \multicolumn{5}{|l|}{ Influenza strain } \\
\hline A Total & $128(64 \cdot 6)$ & $216(69 \cdot 0)$ & $113(49 \cdot 3)$ & $457(61 \cdot 8)$ \\
\hline A/H1N1pdm09 & 83 & 2 & 11 & 96 \\
\hline $\mathrm{A} / \mathrm{H} 3 \mathrm{~N} 2$ & 22 & 77 & 13 & 112 \\
\hline A/untyped & 23 & 137 & 89 & 249 \\
\hline B & $70(35 \cdot 4)$ & $97(31 \cdot 0)$ & $116(50 \cdot 7)$ & $283(38 \cdot 2)$ \\
\hline \multicolumn{5}{|l|}{ Presentation† $\dagger$} \\
\hline Influenza-like illness & $148(78 \cdot 8)$ & $247(79 \cdot 2)$ & $159(69 \cdot 4)$ & $554(74 \cdot 9)$ \\
\hline Fever without source & $17(8 \cdot 5)$ & $24(7 \cdot 7)$ & $20(8 \cdot 7)$ & $61(8 \cdot 2)$ \\
\hline Pneumonia/respiratory distress & $38(19 \cdot 1)$ & $69(22 \cdot 1)$ & $34(14 \cdot 8)$ & $141(19 \cdot 1)$ \\
\hline \multicolumn{5}{|l|}{ Investigations } \\
\hline Chest $X$ ray & $108(54 \cdot 3)$ & $179(57 \cdot 4)$ & $143(62 \cdot 4)$ & $430(58 \cdot 1)$ \\
\hline Lumbar punctures & $31(15 \cdot 6)$ & $31(9 \cdot 9)$ & $28(12 \cdot 2)$ & $90(12 \cdot 2)$ \\
\hline
\end{tabular}

Absolute numbers indicated with percentage within stated year in parentheses unless indicated otherwise.

* $P=0.003$ on binomial testing.

$\dagger$ Individual categories are not mutually exclusive and totals may not equal the sum of all categories.

was the most common $(3 \cdot 0 \%)$ culture-positive bacterial infection (Table 3); bacterial meningitis was rare (one case from Streptococcus pneumoniae in an 11-month-old). Bacteraemia was present in 1.8\% $(n=13)$ of patients and radiologically confirmed pneumonia in $11 \cdot 2 \%(n=83$, including three children with associated bacteraemia). Febrile seizures occurred in $5.9 \%(n=44)$ of children, and $4 \cdot 1 \%(n=30)$ had an afebrile seizure $(23 / 30$ had an underlying seizure disorder). Eleven children had influenza-associated encephalopathy (Table 3). 


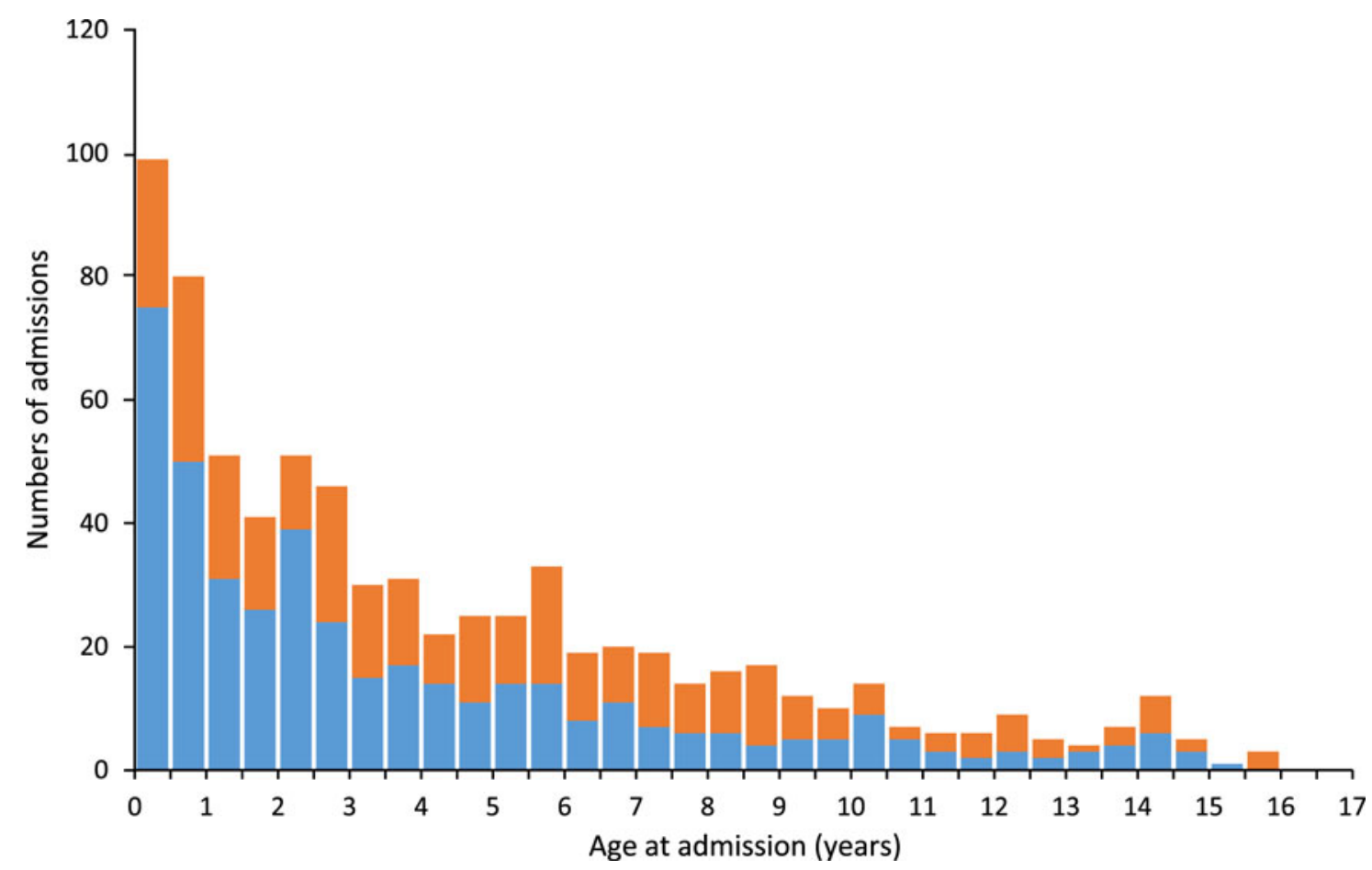

Fig. 1. Age distribution of hospitalized influenza patients $(n=740)$ in Australian children with laboratory-confirmed influenza during 2011-2013 at the Children's Hospital at Westmead (NSW, Australia) and Princess Margaret Hospital (WA, Australia). Numbers of admissions by 6-month age cohorts. Blue bars indicate the proportion of admissions which were healthy children and red bars the proportion with underlying medical conditions.

Table 2. Population-level estimates of influenza vaccine coverage for Australia, New South Wales, and Western Australia for at least one dose of influenza vaccine during 2011-2013 seasons in children aged 6 months to $<7$ years.

\begin{tabular}{lccc}
\hline \hline Jurisdiction & 2011 & 2012 & 2013 \\
\hline Australia & $1 \cdot 0 \%$ & $1 \cdot 0 \%$ & $1 \cdot 6 \%$ \\
New South Wales & $0 \cdot 5 \%$ & $0 \cdot 6 \%$ & $1 \cdot 0 \%$ \\
Western Australia & $3 \cdot 9 \%$ & $3 \cdot 6 \%$ & $5 \cdot 1 \%$ \\
\hline \hline
\end{tabular}

Data obtained from the Australian Childhood Immunisation Register.

\section{Investigations, treatment and outcomes}

Investigations, treatment and outcomes are shown in Tables 1 and 3. Antibiotics were used in $72 \cdot 3 \%$ of patients, while antivirals were prescribed to $20.5 \%$ of patients, a median of 2 days after admission (Table 3); use of antiviral therapy declined over the study period. Admission to ICU was required in $8 \cdot 5 \%(n=63$, including two nosocomial infections), with a median LOS of 3 days [interquartile range (IQR) 2-6 days]. The majority (51/63) of ICU admissions occurred within $24 \mathrm{~h}$ of hospital admission. Of
474 children with vaccination status confirmed by ACIR, 36 were admitted to ICU, none of whom were vaccinated. No vaccinated child $(n=12$ in total) required ICU admission. Multivariate analysis showed cardiac and neurological comorbidities, and diabetes were significantly associated with ICU admission (Table 4). In addition, bacterial co-infection, CXR abnormality, and encephalopathy were also strong predictors of need for ICU admission. Vaccination status was not incorporated into the multivariate model for ICU admission as it was not available for all participants and was not significant in univariate analysis as a risk factor in subset analysis (children aged $<7$ years).

The median LOS for all patients admitted with influenza was 2 days (IQR 1-4) (Table 3). Clinical factors significantly associated with LOS are listed in Table 5. Having a febrile seizure was associated with a shorter LOS. Two children, both aged 8 years and with influenza type A, died during their hospitalization.

\section{DISCUSSION}

This study provides extensive detail on the characteristics and outcomes of laboratory-confirmed 
Table 3. Outcomes and complications in Australian children hospitalized with laboratory-confirmed influenza during 2011-2013 at the Children's Hospital at Westmead (NSW, Australia) and Princess Margaret Hospital (WA, Australia) by year

\begin{tabular}{|c|c|c|c|c|}
\hline & \multicolumn{3}{|l|}{ Year } & \multirow[b]{2}{*}{ Total } \\
\hline & 2011 & 2012 & 2013 & \\
\hline Total $(n)$ & 199 & 312 & 229 & 740 \\
\hline \multicolumn{5}{|l|}{ Outcomes } \\
\hline Median length of stay, days (IQR) & $3(2-6)$ & $2(1-4)$ & $2(1 \cdot 5-4)$ & $2(1-4)$ \\
\hline ICU admission & $15(7 \cdot 5)$ & $27(8 \cdot 7)$ & $21(9 \cdot 2)$ & $63(8 \cdot 5)$ \\
\hline Median ICU length of stay, days (IQR) & $3(2-4)$ & $2(1-4)$ & $4(2-7)$ & $3(2-6)$ \\
\hline Antibiotics prescribed & $147(73 \cdot 9)$ & $221(70 \cdot 8)$ & $167(72 \cdot 9)$ & $535(72 \cdot 3)$ \\
\hline Antivirals prescribed & $54(27 \cdot 1)^{*}$ & $58(18 \cdot 6)$ & $40(17 \cdot 5)^{*}$ & $152(20 \cdot 5)$ \\
\hline \multicolumn{5}{|l|}{ Complications } \\
\hline Bacterial co-infection & $27(13 \cdot 6)$ & $31(9 \cdot 9)$ & $33(14 \cdot 4)$ & $91(12 \cdot 3) \dagger$ \\
\hline Meningitis & $1(0 \cdot 5)$ & 0 & 0 & $1(0 \cdot 1)$ \\
\hline Bacteraemia & $5(2 \cdot 5)$ & $2(0 \cdot 6)$ & $6(2 \cdot 6)$ & $13(1 \cdot 8)$ \\
\hline Urinary tract infection & $4(2 \cdot 0)$ & $8(2 \cdot 6)$ & $10(4 \cdot 4)$ & $22(3 \cdot 0)$ \\
\hline Other & $19(9 \cdot 5)$ & $22(7 \cdot 1)$ & $19(8 \cdot 3)$ & $60(8 \cdot 1)$ \\
\hline Viral co-infection & $43(21 \cdot 6)$ & $47(15 \cdot 1)$ & $40(17 \cdot 5)$ & $130(17 \cdot 6)$ \\
\hline Pneumonia/pneumonitis & $24(12 \cdot 1)$ & $40(12 \cdot 8)$ & $19(8 \cdot 3)$ & $83(11 \cdot 2)$ \\
\hline Seizures & $20(10 \cdot 1)$ & $31(9 \cdot 9)$ & $23(10 \cdot 0)$ & $74(10 \cdot 0)$ \\
\hline Febrile seizures & 10 & 21 & 13 & $44(5 \cdot 9)$ \\
\hline Afebrile seizures & 10 & 10 & 10 & $30(4 \cdot 1)$ \\
\hline Encephalopathy/encephalitis & $3(1 \cdot 5)$ & $5(1 \cdot 6)$ & $3(1 \cdot 3)$ & $11(1 \cdot 5)$ \\
\hline Myositis & 0 & $4(1 \cdot 3)$ & $7(3 \cdot 1)$ & $11(1 \cdot 5)$ \\
\hline Death & $1(0 \cdot 5)$ & 0 & $1(0 \cdot 4)$ & $2(0 \cdot 3)$ \\
\hline
\end{tabular}

IQR, Interquartile range; ICU, intensive care unit.

Absolute numbers indicated with percentage within stated year in parentheses unless indicated otherwise.

* $P=0.02$ using $\chi^{2}$ test.

$\uparrow$ Total bacterial co-infection included individuals with $\geqslant 1$ co-infection and totals may not equal the sum of all categories.

Table 4. Risk factors associated with intensive care unit admission in Australian children hospitalized with laboratory-confirmed influenza during 2011-2013 at the Children's Hospital at Westmead (NSW, Australia) and Princess Margaret Hospital (WA, Australia)

\begin{tabular}{llclc}
\hline \hline Predictor variable & $\begin{array}{l}\text { Unadjusted OR } \\
(95 \% \text { CI })\end{array}$ & $P$ value & $\begin{array}{l}\text { Adjusted multivariate OR } \\
(95 \% \text { CI })\end{array}$ & $P$ value \\
\hline Respiratory comorbidity & $1 \cdot 96(0 \cdot 97-3 \cdot 94)$ & $0 \cdot 06$ & NI & NI \\
Cardiac comorbidity & $4 \cdot 19(2 \cdot 05-8 \cdot 55)$ & $<0 \cdot 001$ & $3 \cdot 83(1 \cdot 74-8 \cdot 45)$ & $0 \cdot 001$ \\
Diabetes & $4 \cdot 41(0 \cdot 84-23 \cdot 2)$ & $0 \cdot 08$ & $7 \cdot 44(1 \cdot 11-49 \cdot 7)$ & $0 \cdot 04$ \\
Neurological comorbidity & $3 \cdot 79(2 \cdot 12-6 \cdot 79)$ & $<0 \cdot 001$ & $2 \cdot 15(1 \cdot 05-4 \cdot 40)$ & $0 \cdot 04$ \\
Immunosuppression & $0 \cdot 20(0 \cdot 05-0 \cdot 83)$ & $0 \cdot 03$ & NI & NI \\
Genetic comorbidity & $2 \cdot 52(1 \cdot 17-5 \cdot 45)$ & $0 \cdot 19$ & NI & $0 \cdot 01$ \\
Bacterial co-infection & $3 \cdot 31(1 \cdot 82-6 \cdot 02)$ & $<0 \cdot 001$ & $2 \cdot 41(1 \cdot 23-4 \cdot 73)$ & NI \\
Viral co-infection & $1 \cdot 66(0 \cdot 91-3 \cdot 04)$ & $0 \cdot 10$ & NI & $0 \cdot 01$ \\
Afebrile seizure & $7 \cdot 32(3 \cdot 31-7 \cdot 33)$ & $<0 \cdot 001$ & $3 \cdot 71(1 \cdot 34-10 \cdot 3)$ & $<0 \cdot 001$ \\
CXR abnormality & $3 \cdot 53(2 \cdot 05-6 \cdot 06)$ & $<0 \cdot 001$ & $3 \cdot 75(2 \cdot 06-6 \cdot 83)$ & $0 \cdot 002$ \\
Encephalitis/encephalopathy & $9 \cdot 64(2 \cdot 85-32 \cdot 5)$ & $<0 \cdot 001$ & $10 \cdot 78(2 \cdot 43-47 \cdot 7)$ & \\
\hline \hline
\end{tabular}

OR, Odds ratio; CI, confidence interval; NI, variable not included in final model.

Unadjusted odds ratios calculated from univariate analysis of predictors, with variables with $P<0 \cdot 20$ listed and incorporated into a backwards stepwise multivariate logistic regression model. 
Table 5. Risk factors associated with length of stay in Australian children hospitalized with laboratory-confirmed influenza during 2011-2013 at the Children's Hospital at Westmead (NSW, Australia) and Princess Margaret Hospital (WA, Australia) excluding nosocomial cases with onset of symptoms $>7$ days after admission

\begin{tabular}{|c|c|c|c|c|}
\hline Predictor variable & $\begin{array}{l}\text { Unadjusted OR of } \\
\text { means }(95 \% \mathrm{CI})\end{array}$ & $P$ value & $\begin{array}{l}\text { Adjusted OR of } \\
\text { means }(95 \% \mathrm{CI})\end{array}$ & $P$ value \\
\hline Female sex & $1.27(1.08-1.49)$ & 0.004 & $1.22(1.02-1.44)$ & 0.03 \\
\hline CXR abnormality & $1.65(1.40-1.95)$ & $<0 \cdot 001$ & $1.50(1.25-1 \cdot 79)$ & $<0 \cdot 001$ \\
\hline Bacterial co-infection & $2 \cdot 31(1 \cdot 83-2 \cdot 92)$ & $<0.001$ & $2.46(1.93-3.14)$ & $<0 \cdot 001$ \\
\hline Viral co-infection & $1.64(1.34-2.01)$ & $<0.001$ & $1.72(1.38-2.15)$ & $<0 \cdot 001$ \\
\hline Febrile seizures & $0.48(0.33-0.68)$ & $<0 \cdot 001$ & $0.62(0.42-0.89)$ & $0 \cdot 01$ \\
\hline Afebrile seizure & $1 \cdot 64(1 \cdot 10-2 \cdot 44)$ & $0 \cdot 02$ & NI & NI \\
\hline Encephalitis/encephalopathy & $1 \cdot 56(0 \cdot 80-3 \cdot 03)$ & $0 \cdot 19$ & $2 \cdot 47(1 \cdot 24-4 \cdot 90)$ & $0 \cdot 01$ \\
\hline Respiratory comorbidity & $1.58(1.22-2 \cdot 03)$ & $<0 \cdot 001$ & $1.40(1.06-1.84)$ & 0.02 \\
\hline Prematurity & $1 \cdot 54(1 \cdot 17-2 \cdot 02)$ & 0.002 & $1.45(1.08-1.94)$ & $0 \cdot 01$ \\
\hline Cardiac comorbidity & $1.38(1.01-1.89)$ & $0 \cdot 05$ & NI & NI \\
\hline Diabetes & $1 \cdot 75(0 \cdot 80-3 \cdot 85)$ & $0 \cdot 16$ & $2 \cdot 22(1 \cdot 00-4 \cdot 96)$ & 0.05 \\
\hline Renal comorbidity & $1.60(1.04-2.47)$ & 0.03 & $1.89(1.20-2.98)$ & $0 \cdot 006$ \\
\hline Hepatic comorbidity & $3 \cdot 91(2 \cdot 39-6 \cdot 42)$ & $<0 \cdot 001$ & $5 \cdot 60(3 \cdot 37-9 \cdot 31)$ & $<0 \cdot 001$ \\
\hline Genetic comorbidity & $1 \cdot 63(1 \cdot 20-2 \cdot 23)$ & 0.002 & $1.45(1.04-2.03)$ & $0 \cdot 03$ \\
\hline Metabolic comorbidity & $1 \cdot 48(0 \cdot 84-2 \cdot 60)$ & $0 \cdot 17$ & NI & NI \\
\hline
\end{tabular}

OR, Odds ratio; CI, confidence interval; NI, variable not included in final model after backwards stepwise elimination. Generalized linear regression model with negative binomial distribution. Predictor variables with $P<0 \cdot 20$ on univariate analysis listed and incorporated into a final multivariate model.

hospitalized influenza in Australian children over a 3 -year period in two geographically distinct areas. While the relative over-representation of young children aged $<5$ years is consistent with administrative data $[1,3]$, this study provides new insight into the clinical characteristics of children requiring hospitalization. Although $43 \%$ of children had underlying medical conditions, the majority were healthy. Infants aged 0-6 months represented the highest proportion (by 6-month age cohorts) of hospitalized children in our study; this age group is too young for influenza vaccination, but may be afforded protection from maternal vaccination $[29,30]$.

Children hospitalized with influenza had a rate of complications comparable to adults, including pneumonia and seizures in $1 / 10$, with ICU admission required in 1/12 children, similar to reports from seasonal and pandemic years [22, 31, 32]. Despite the median LOS being shorter than in adults, these findings confirm that influenza should not be regarded as a benign paediatric illness. Neurological complications such as acute encephalitis were present in a minority $(n=11,1.5 \%)$ but most cases were in previously healthy children and encephalitis was a strong predictor of ICU admission. Influenza has since been confirmed as a significant preventable cause of childhood encephalitis $(10 \%)$ in a prospective multi- centre Australian study of acute encephalitis [33]. Other risk factors for ICU admission included cardiac, neurological disease, and diabetes, consistent with systematic reviews which found these factors to be predictors of severe influenza [34, 35].

Our study revealed high rates of potentially unnecessary antibiotic use in hospitalized influenza cases and also the infrequent and progressively declining use of antiviral medications (27\% in 2011 to $18 \%$ in 2013). Prospectively collected FluCAN data from 2014 has shown this to fall further to $15 \%$ [23] and demonstrates the consistent decline in utilization rates from a high of around $50 \%$ during the 2009 pandemic [36] It is likely that with increasing time since the 2009 pandemic, physician prescribing may be falling back to pre-pandemic levels, when antiviral prescription was relatively uncommon [37].

The cost to the health system of paediatric influenza hospitalization is likely to be considerable but it is difficult to quantify. A recent analysis used weighted costings for the most frequent Australian Refined Diagnosis Related Group (AR-DRG) codes associated with individual national hospitalization data [38] where influenza was coded as the primary diagnosis, and estimated \$3473-3560 cost per admission (2016 AU\$) for children aged 0-17 years [39]. Applying this cost estimate per episode to our study 
$(n=740)$, the annual direct cost would be AU\$ 850000 per year for the two hospitals alone. The cost burden on the health system is likely to be high, particularly given the under-recognition of influenza as a cause of many hospitalizations [20].

Influenza vaccine uptake was low in all children hospitalized with influenza (5\% in those with medical conditions and $0.7 \%$ in those without). The higher uptake in PMH $(4 \cdot 3 \%)$ compared to CHW $(1 \cdot 6 \%)$ suggests the availability and promotion of free vaccine for children aged 6 months to $<5$ years in the state of WA was a factor in vaccine uptake, albeit still only having a modest impact on coverage. Hospitalization of a child increased vaccination uptake in the season following each child's hospitalization to $10 \cdot 8 \%$, possibly due to increased awareness by parents of the risk and morbidity of childhood influenza. While this is promising, there remain unanswered questions as to why this figure remains low and how it could be further improved. Increased recommendation of vaccination and addressing of any parental safety concerns at the time of hospitalization by treating physicians may be strategies worthy of consideration.

While our study was limited to directly examining coverage in a cohort of hospitalized children, in whom rates may be lower due to vaccine effectiveness preventing hospitalization, we also found state and national population-level estimates to be very similar. Importantly, our data provides evidence of the low vaccination uptake in children with comorbidities, information which is not collected by the national ACIR. Our study also informed the establishment of prospective active surveillance in specialist paediatric hospitals (annually since the 2014 season) to complement existing FluCAN surveillance in adult and general centres. This prospective surveillance has recently reported on vaccine coverage and effectiveness in the 2014 season [23], and together with a second longitudinal study in WA [40] confirmed low vaccine coverage in hospitalized children, including influenza testnegative ('control') populations, in whom uptake was only $8 \cdot 5-12 \cdot 4 \%$ overall and $18 \%$ for children with comorbidities. The true national population rate is likely to be lower, particularly considering both studies had recruitment of children in WA. These estimates all point to worryingly low influenza vaccination coverage in at-risk children who have been eligible for free vaccination at point-of-care since 2010 .

Our data suggests that improving vaccination coverage should be a focus for reduction of influenza morbidity, particularly in those with underlying medical conditions. In addition, the absence of strong recommendations and national funding for vaccination of healthy children means a significant population within the community are unprotected and contribute to the burden of influenza hospitalization nationally. With the knowledge that hospitalization rates in Australia are highest in those aged $<5$ years $[1,3]$, the ongoing poor coverage in WA, despite its partially funded ( 6 months $-<5$ years) programme for all children is disappointing, but indicates the sustained impact of vaccine safety concerns after elevated post-vaccination febrile seizure rates were seen from one vaccine brand in 2010. Following this, vaccine uptake in WA fell from approximately 45\% (2008 and 2009) to 7\% during (2011-2015) despite continued funding, as shown in this and other studies [10]; the proportion of parents who felt influenza vaccine to be safe halved [10]. Universally recommended and funded influenza vaccination, as currently provided in numerous countries, has the potential to increase public confidence in influenza vaccine safety and uptake in all children. Coverage of approximately $60 \%$ has been achieved in the United States' [17] and UK's universal childhood vaccination programmes [18] and has resulted in reductions for a range of influenza-related outcomes both directly in targeted age groups and indirectly via herd protection in unimmunized age groups [18, 41, 42].

Limitations in this retrospective study were that data collection was restricted to information available from case records. Vaccination status was poorly recorded in clinical notes and it is clear that specific medical history taking about influenza vaccination status should become common practice. We confined our analysis to PCR-confirmed influenza only, for consistency across both sites, which together with probable under-ascertainment of cases due to nonspecific presentations and likely incomplete testing, suggests the total influenza burden was underestimated. While respiratory virus testing on hospitalized patients with respiratory illness is routinely recommended for infection control purposes, it was ultimately at the physician's discretion and we could not be certain that all such patients were tested. A strength of our study, is that we have obtained data on influenza vaccine uptake in at-risk populations, albeit influenza cases, but have related that to state and national estimates, and estimates in hospitalized noninfluenza patients.

In summary, this study provides detailed vaccination and clinical data on Australian children 
hospitalized with influenza. It demonstrates that influenza has a significant impact on young children, both healthy and with underlying medical conditions, and can be associated with significant morbidity. There is very low vaccination coverage in this hospitalized cohort, which matches low estimates of coverage at a population level and in non-influenza hospitalized children. Funded immunisation programmes for at-risk children, and in WA for some healthy children, are not achieving good vaccine uptake; this strongly suggests the need to examine additional strategies. Accurate local disease modelling to investigate future options, such as universal free influenza vaccination in children, and other studies examining barriers to vaccine uptake, would be valuable.

\section{ACKNOWLEDGEMENTS}

We thank the FluCAN team at The Children's Hospital at Westmead: Laura Rost, Jocelynne McRae, Corina Shiels and Deepika Mahajan; and at Princess Margaret Hospital: Carolyn Finucane, and Heidi Hutton; Liz Barnes for statistical advice; Janine Roney and Jill Garlick for central support. The Departments of Health in Western Australia and New South Wales provided data on numbers of influenza hospitalization.

The Australian Department of Health provided funding for this study and provides ongoing support for the FluCAN surveillance system. Chris Blyth is supported by a WA Health/Raine Clinical Research Fellowship and a NHMRC Career Development Fellowship (APP1111596). Allen Cheng is supported by an NHMRC Career Development Fellowship.

\section{DECLARATION OF INTEREST}

None.

\section{REFERENCES}

1. Chiu C, et al. Vaccine preventable diseases in Australia, 2005 to 2007. Communicable Diseases Intelligence 2010; 34 (Suppl.): S1-167.

2. Chiu C, Wang H, Macartney K. Influenza hospitalisations in young Australian Children during seasonal and pandemic periods. Communicable Disease Control (CDC) Conference, Canberra, Australia, 2013.

3. Li-Kim-Moy J, et al. Australian vaccine preventable disease epidemiological review series: Influenza 2006-2013. Communicable Diseases Intelligence 2016; 40: E482-E495.

4. Poehling KA, et al. The burden of influenza in young children, 2004-2009. Pediatrics 2013; 131: 207-216.
5. Australian Technical Advisory Group on Immunisation (ATAGI). Clinical advice for immunisation providers regarding the administration of 2015 seasonal influenza vaccines. 2015 (http://www.immunise.health.gov.au/ internet/immunise/publishing.nsf/content/atagi-advice-tiv). Accessed 6 December 2015.

6. Department of Health, Australia. Free flu vaccines for Indigenous children (http://www.health.gov.au/internet/ ministers/publishing.nsf/Content/health-mediarel-yr2015ley018.htm). Accessed 9 June 2015.

7. Government of Western Australia, Department of Health. Paediatric influenza immunisation: the 2008 metropolitan trial 2008 (http://ww2.health.wa.gov.au/ /media/Files/ Corporate/general\%20documents/Infectious\%20diseases/ PDF/DWAtch/DWatch2008vol12.1.ashx). Accessed 14 August 2016.

8. Armstrong PK, et al. Epidemiological study of severe febrile reactions in young children in Western Australia caused by a 2010 trivalent inactivated influenza vaccine. BMJ Open 2011; 1: e000016.

9. Therapeutic Goods Administration. Overview of vaccine regulation and safety monitoring and investigation into adverse events following 2010 seasonal influenza vaccination in young children 2010 (https://www.tga.gov.au/ file/555/download). Accessed 11 September 2012.

10. Blyth CC, et al. The impact of pandemic $\mathrm{A}(\mathrm{H} 1 \mathrm{~N} 1)$ pdm09 influenza and vaccine-associated adverse events on parental attitudes and influenza vaccine uptake in young children. Vaccine 2014; 32: 4075-4081.

11. Wood N, et al. Influenza vaccine safety in children less than 5 years old: the 2010 and 2011 experience in Australia. Pediatric Infectious Disease Journal 2012; 31: 199-202.

12. Pillsbury A, et al. Real-time safety surveillance of seasonal influenza vaccines in children, Australia, 2015. Eurosurveillance 2015; 20(43).

13. National Advisory Committee on Immunization (NACI). Statement on seasonal influenza vaccine for 2015-2016. 2015 (http://www.phac-aspc.gc.ca/naci-ccni/flu-2015grippe-eng.php). Accessed 19 April 2016.

14. Department of Health, England, UK. Flu plan: winter 2015 to 2016 (https://www.gov.uk/government/publications/flu-plan-2015-to-2016). Accessed 27 August 2015.

15. Grohskopf LA, et al. Prevention and control of influenza with vaccines: recommendations of the Advisory Committee on Immunization Practices, United States, 2015-16 influenza season. Morbidity and Mortality Weekly Report 2015; 64: 818-825.

16. Government of Canada. Public funding for influenza vaccination by province/territory (as of July 2016) (http:// healthycanadians.gc.ca/healthy-living-vie-saine/immunization-immunisation/schedule-calendrier/influenza-vaccination-grippe-eng.php). Accessed 19 April 2016.

17. Centers for Disease Control and Prevention. Flu vaccination coverage, United States, 2014-15 influenza season. 2015 (http://www.cdc.gov/flu/fluvaxview/coverage1415estimates.htm). Accessed 8 December 2015.

18. Pebody RG, et al. Uptake and impact of vaccinating school age children against influenza during a season with circulation of drifted influenza A and B strains, England, 2014/15. Eurosurveillance 2015; 20: pii $=30029$. 
19. Newcombe J, et al. Prevalence and determinants of influenza vaccine coverage at tertiary pediatric hospitals. Vaccine 2014; 32: 6364-6368.

20. Beard F, et al. Influenza related hospitalisations in Sydney, New South Wales, Australia. Archives of Disease in Childhood 2006; 91: 20-25.

21. Iskander M, et al. The burden of influenza in children under 5 years admitted to the Children's Hospital at Westmead in the winter of 2006. Journal of Paediatrics and Child Health 2009; 45: 698-703.

22. Khandaker G, et al. Clinical epidemiology and predictors of outcome in children hospitalised with influenza A(H1N1)pdm09 in 2009: a prospective national study. Influenza and Other Respiratory Viruses 2014; 8: 636645.

23. Blyth CC, et al. Influenza epidemiology, vaccine coverage and vaccine effectiveness in children admitted to sentinel Australian hospitals in 2014: the Influenza Complications Alert Network (FluCAN). Eurosurveillance 2016; 21: pii=30301.

24. Kelly PM, et al. FluCAN 2009: initial results from sentinel surveillance for adult influenza and pneumonia in eight Australian hospitals. Medical Journal of Australia 2011; 194: 169-174.

25. Australian Government Department of Health. The Australian Immunisation Handbook, 10th edn, 2015 (http://www.immunise.health.gov.au/internet/immunise/ publishing.nsf/Content/7B28E87511E08905CA257D4D 001DB1F8/\$File/Aus-Imm-Handbook.pdf). Accessed 2 February 2016.

26. Australian Government Department of Human Services. Australian Childhood Immunisation Register (https:// www.humanservices.gov.au/customer/services/medicare/ australian-childhood-immunisation-register). Accessed 18 August 2016.

27. Australian Bureau of Statistics. Births, Australia, 2014. Canberra 2014 (http://www.abs.gov.au/ausstats/abs@. nsf/mf/3301.0). Accessed 18 December 2015.

28. Centers for Disease Control and Prevention. Overview of influenza surveillance in the United States (http://www. cdc.gov/flu/weekly/overview.htm). Accessed 21 August 2016.

29. Madhi SA, et al. Influenza vaccination of pregnant women and protection of their infants. New England Journal of Medicine 2014; 371: 918-931.

30. Dabrera G, et al. Effectiveness of seasonal influenza vaccination during pregnancy in preventing influenza infection in infants, England, 2013/14. Eurosurveillance 2014; 19: 20959.
31. Khandaker G, et al. Pandemic (H1N1) 2009 and seasonal influenza A (H3N2) in Children's Hospital, Australia. Emerging Infectious Diseases 2011; 17: 1960-1962.

32. Australian Government Department of Health. Australian influenza surveillance report: 26 September to 9 October 2015. 2015 (http://www.health.gov.au/internet/main/ publishing.nsf/Content/cda-surveil-ozflu-flucurr.htm/\$File/ Australian-Influenza-Surveillance-Report.pdf). Accessed 7 April 2016.

33. Britton $\mathbf{P}$, et al. Influenza-associated neurological disease: cases identified by the Australian Childhood Encephalitis (ACE) Study 2015 (https://idsa.confex.com/idsa/2015/ webprogram/Paper52981.html). Accessed 18 December 2015.

34. Gill PJ, et al. Identification of children at risk of influenza-related complications in primary and ambulatory care: a systematic review and meta-analysis. Lancet Respiratory Medicine 2015; 3: 139-149.

35. Mertz D, et al. Populations at risk for severe or complicated influenza illness: systematic review and meta-analysis. British Medical Journal (Clinical Research Edition) 2013; 347: f5061.

36. Elliott EJ, et al. Novel inpatient surveillance in tertiary paediatric hospitals in New South Wales illustrates impact of first-wave pandemic influenza A H1N1 (2009) and informs future health service planning. Journal of Paediatrics and Child Health 2012; 48: 235241.

37. Lester-Smith D, et al. The burden of childhood influenza in a tertiary paediatric setting. Communicable Diseases Intelligence 2009; 33: 209-215.

38. Australian Institute of Health and Welfare. National hospital morbidity database (NHMD) (http://www.aihw.gov. au/hospitals-data/national-hospital-morbidity-database/).

39. Yin JK, et al. Influenza-associated hospitalizations in Australia: estimated health and economic burden (poster). Options IX for the Control of Influenza, 24-28 August 2016, Chicago, USA. 2016.

40. Blyth CC, et al. The effectiveness of influenza vaccination in preventing hospitalisation in children in Western Australia. Vaccine 2015; 33: 7239-7244.

41. Pebody R, et al. Effectiveness of seasonal influenza vaccine in preventing laboratory-confirmed influenza in primary care in the United Kingdom: 2014/15 end of season results. Eurosurveillance 2015; 20(36).

42. Pebody RG, et al. Uptake and impact of a new live attenuated influenza vaccine programme in England: early results of a pilot in primary school-age children, 2013/ 14 influenza season. Eurosurveillance 2014; 19(22). 